\title{
Behaviour of a two-planetary system on a cosmogonic time-scale
}

\author{
Konstantin V. Kholshevnikov ${ }^{1}$ \\ and Eduard D. Kuznetsov ${ }^{2}$ \\ ${ }^{1}$ Sobolev Astronomical Institute, St.Petersburg State University, Universitetsky pr., 28, \\ St.Petersburg, Stary Peterhof, 198504 Russia \\ email: kvk@astro.spbu.ru \\ ${ }^{2}$ Astronomical Observatory, Urals State University, Lenin pr., 51, Ekaterinburg, 620083 Russia \\ email: Eduard.Kuznetsov@usu.ru
}

\begin{abstract}
The orbital evolution of planetary systems similar to our Solar one represents one of the most important problems of Celestial Mechanics. In the present work we use Jacobian coordinates, introduce two systems of osculating elements, construct the Hamiltonian expansions in Poisson series for all the elements for the planetary three-body problem (including the problem Sun-Jupiter-Saturn). Further we construct the averaged Hamiltonian by the Hori-Deprit method with accuracy up to second order with respect to the small parameter, the generating function, the change of variables formulae, and the right-hand sides of the averaged equations. The averaged equations for the Sun-Jupiter-Saturn system are integrated numerically over a time span of 10 Gyr. The Liapunov Time turns out to be 14 Myr (Jupiter) and 10 Myr (Saturn).
\end{abstract}

Keywords. Celestial mechanics, methods: analytical, methods: numerical, planets and satellites, Jupiter, Saturn

\section{The Hamiltonian of the planetary three-body problem}

The orbital evolution of a planetary system similar to our Solar one represents one of the most important problems of Celestial Mechanics. The secular behaviour of the planetary three-body problem has been extensively investigated both from the mathematical and numerical point of view by many researchers. The stability of the spatial planetary three-body problem using KAM theory has been investigated by Robutel (1993a), Robutel (1993b), Laskar \& Robutel (1995), Robutel (1995). These results have been obtained for extremely small planetary eccentricities and masses. The motion of the Jupiter-Saturn planetary system near the $5: 2$ mean-motion resonance has been modeled analytically by Michtchenko \& Ferraz-Mello (2001) in the framework of the planar general three-body problem.

In this paper, we continue researches of the spatial planetary three-body problem Sun-Jupiter-Saturn begun in Kholshevnikov, Greb, \& Kuznetsov (2001), Kholshevnikov, Greb, \& Kuznetsov (2002) and Kuznetsov \& Kholshevnikov (2004). We use Jacobian coordinates as the best suited. Let us assume $m_{0}, \mu m_{0} m_{1}, \mu m_{0} m_{2}$ as masses of the Sun, Jupiter and Saturn respectively. The small parameter $\mu$ is equal to $10^{-3}$. In this case the dimensionless masses $m_{1}$ and $m_{2}$ are of order unity $\left(m_{1} \approx 1, m_{2} \approx 1 / 3\right)$.

Let us represent the Hamiltonian as a sum of the unperturbed part $h_{0}$ and the perturbed one $\mu h_{1}$ :

$$
h=h_{0}+\mu h_{1}
$$


The first term depends on the semi-major axes only

$$
h_{0}=-\frac{G m_{0} m_{1}}{2 a_{1}}-\frac{G m_{0} m_{2}}{2 a_{2}},
$$

$G$ being the gravitational constant. Here and below the subscripts 1 and 2 for coordinates and elements correspond to Jupiter and Saturn respectively. The second term of (1.1) may be thought of as a constant factor having the dimension of velocity squared and a dimensionless part $h_{2}$

$$
h_{1}=\frac{G m_{0}}{a_{0}} h_{2}, \quad h_{2}=h_{3}+h_{4},
$$

where $a_{0}$ is an arbitrary parameter with dimension of the length,

$$
\begin{gathered}
h_{3}=\frac{m_{2} a_{0}}{\mu}\left(\frac{1}{r_{2}}-\frac{1}{\rho}\right)=\frac{m_{2} a_{0}\left[2 \frac{m_{1}}{1+\mu m_{1}} \mathbf{r}_{1} \mathbf{r}_{2}+\mu\left(\frac{m_{1}}{1+\mu m_{1}}\right)^{2} r_{1}^{2}\right]}{r_{2} \rho\left(r_{2}+\rho\right)}, \\
h_{4}=-\frac{m_{1} m_{2} a_{0}}{\Delta}, \quad \rho=\left|\mathbf{r}_{2}+\frac{\mu m_{1}}{1+\mu m_{1}} \mathbf{r}_{1}\right|, \quad \Delta=\left|\mathbf{r}_{2}-\frac{1}{1+\mu m_{1}} \mathbf{r}_{1}\right| .
\end{gathered}
$$

\section{Systems of osculating elements}

Let us introduce two systems of osculating elements. The first system is close to the Keplerian one

$$
x_{3 s-2}^{(1)}=\widetilde{a}_{s}, \quad x_{3 s-1}^{(1)}=e_{s}, \quad x_{3 s}^{(1)}=\widetilde{I}_{s}, \quad y_{3 s-2}^{(1)}=\alpha_{s}, \quad y_{3 s-1}^{(1)}=\beta_{s}, \quad y_{3 s}^{(1)}=\gamma_{s} .
$$

Here $\widetilde{a}=\left(a-a^{0}\right) / a^{0}, \widetilde{I}=\sin (I / 2), \alpha=l+g+\Omega, \beta=g+\Omega, \gamma=\Omega$ are expressed in terms of Keplerian elements $a, a^{0}, e, I, l, g, \Omega$ : semi-major axis and its mean value, eccentricity, inclination, mean anomaly, argument of pericenter, longitude of ascending node. The index $s$ changes from 1 to the number of planets $N=2$.

The second system realizes simplifications due to the homogeneity of the perturbation function with respect to the semi-major axes. In this system the denominators arising in a process of averaging transforms are extremely simple. On the other hand, it introduces a complication, by mixing the orbital elements of different planets

$$
x_{3 s-2}^{(2)}=z_{s}, \quad x_{3 s-1}^{(2)}=e_{s}, \quad x_{3 s}^{(2)}=\widetilde{I}_{s}, \quad y_{3 s-2}^{(2)}=\alpha_{s}, \quad y_{3 s-1}^{(2)}=\beta_{s}, \quad y_{3 s}^{(2)}=\gamma_{s},
$$

where $z_{1}=\omega_{1}^{0} / \omega_{1}-1, z_{2}=\left(\omega_{1}^{0} \omega_{2}\right) /\left(\omega_{2}^{0} \omega_{1}\right)-1$. Here $\omega_{s}=\kappa_{s} a_{s}^{-3 / 2}$ are mean motions of the planets, $\omega_{s}^{0}$ are constants close to mean values $\omega_{s}, \kappa_{s}^{2}=G m_{0} m_{s} / M_{s}$ are gravitational parameters of the planets, reduced masses are $M_{s}=m_{s}\left(1+\mu m_{1}+\cdots+\mu m_{s-1}\right) /(1+$ $\left.\mu m_{1}+\cdots+\mu m_{s}\right), s=1,2$.

\section{Expansion of disturbing Hamiltonian into Poisson series}

The disturbing Hamiltonian $h_{2}$ is presented as Poisson series

$$
h_{2}=\sum A_{k n} x^{k} \cos n y .
$$

Here $x=\left\{x_{1}, \ldots, x_{6}\right\}$ are action-like elements, $y=\left\{y_{1}, \ldots, y_{6}\right\}$ are angular ones, $A_{k n}$ are numerical coefficients, $k=\left\{k_{1}, \ldots, k_{6}\right\}$ and $n=\left\{n_{1}, \ldots, n_{6}\right\}$ are multi-indices. The summation is taken over non-negative $k_{s}$ and $n_{1}$ and integer $n_{2}, \ldots, n_{6}$. 
It is well known (see for example Charlier 1927, Subbotin 1968) that

$$
\begin{gathered}
n_{1}+n_{2}+n_{3}+n_{4}+n_{5}+n_{6}=0, \quad n_{3}+n_{6}=\text { even }, \\
k_{s}=\left|n_{s}\right|+\text { non-negative even }(s=2,3,5,6)
\end{gathered}
$$

The simplest restrictions on $k$ and $n$ are

$$
k_{1}+k_{2}+\cdots+k_{6} \leqslant d, \quad n_{1} \leqslant c, \quad\left|n_{4}\right| \leqslant c .
$$

The other $n_{s}$ are less than $d$ according to D'Alembertian properties of the Hamiltonian $h_{2}$ (Kholshevnikov 1997, 2001).

Designate $b$ the order of approximation with respect to $\mu$. To evaluate $d(b)$ it is sufficient to take into account that Jupiter's and Saturn's eccentricities $e_{1}=0.05, e_{2}=0.05$ and the sines of the half-angles of inclinations $I_{1}=0.01, I_{2}=0.02$ are small values of the same order $\mu_{1} \sim \mu^{1 / 2}$. The choice of $c(b)$ is controlled by the rate of convergence at $\mu_{1}=0$. According to Kholshevnikov, Greb, \& Kuznetsov (2002):

$$
d(2)=6, \quad d(3)=11, \quad d(4)=16, \quad c(2)=13, \quad c(3)=25, \quad c(4)=37 .
$$

The parameters $a_{0}, m_{1}, m_{2}, a_{1}^{0}, a_{2}^{0}$ (necessary to calculate the coefficients $A_{k n}$ and the multiplier to convert the Hamiltonian $h_{1}$ ) are given in Kholshevnikov, Greb, \& Kuznetsov (2002). In this paper it is shown also that the expansions up to $\mu^{4}$ has only one small divisor $2 \omega_{1}^{0}-5 \omega_{2}^{0}$. The constant $F=\left|2 \omega_{1}^{0}-5 \omega_{2}^{0}\right| / \omega_{1}^{0}$ describing commensurability degree is equal to 0.023331 for the chosen values of the parameters: this indicates that a weak resonance $F \sim \sqrt{\mu}$ is present.

The Poisson series processor PSP (Brumberg 1995, Ivanova 1996) is used to construct the expansion of disturbing Hamiltonian $h_{2}$ into Poisson series (3.1). The rational version of the PSP is used to decrease round-off errors during calculations of the coefficients $A_{k n}$. The expansion of the disturbing Hamiltonian is processed up to $\mu^{2}$. The summation is taken over $k_{1}+\cdots+k_{6} \leqslant 6,\left|n_{s}\right| \leqslant 15(s=1, \ldots, 6)$. For each of the osculating elements system two variants of the expansion are constructed. The first variant deals with numerical values of parameters (masses, mean values of semi-major axes, ...) corresponding to the Sun-Jupiter-Saturn system. The second one deals with the litteral expressions depending upon the parameters of the system.

\section{Averaged planetary three-body problem}

The Hori-Deprit method (Lie transformation method) is used to construct the averaged Hamiltonian $H$. This method is based on Poisson brackets that allow us to use non-canonical elements, by writing down the Poisson brackets in the corresponding system of phase variables (Kholshevnikov \& Greb 2001).

For a stationary change of variables without mixing impulses $p=\left(p_{1}, \ldots, p_{6}\right)$ and coordinates $q=\left(q_{1}, \ldots, q_{6}\right)$ the Poisson bracket is written down through partial brackets (Kholshevnikov \& Greb 2001) as

$$
\{f, g\}=V_{j k}(f, g)_{j k}, \quad V_{j k}=\frac{\partial x_{j}}{\partial p_{i}} \frac{\partial y_{k}}{\partial q_{i}}, \quad(f, g)_{j k}=\frac{\partial f}{\partial x_{j}} \frac{\partial g}{\partial y_{k}}-\frac{\partial f}{\partial y_{k}} \frac{\partial g}{\partial x_{j}} .
$$

Summation is made by repeating indices from 1 to 6 .

The matrices $\mathcal{V}$ with elements $V_{j k}$ for both the first and the second systems of elements (2.1), (2.2) was obtained in Kholshevnikov \& Greb 2001, Greb (2002). For the first system (2.1) the Poisson matrix is block diagonal. For the second one (2.2) it is triangular. 
The Hamiltonian $h(1.1)$ is averaged over the fast variables $\alpha_{1}$ and $\alpha_{2}$. The averaged Hamiltonian $H$ is represented by a power series in the small parameter $\mu$. The Hamiltonian $H$ and the generating function are echeloned Poisson series (Rom 1971).

For calculations we use the rational version of the echeloned Poisson series processor EPSP (Ivanova 2001) to reduce the round-off errors. Transformations are made for both systems of elements with numerical parameters corresponding to the Sun-Jupiter-Saturn system.

The Hamiltonian $H$ is represented by an echeloned Poisson series upto $\mu^{2}$. Two approximations of Hori-Deprit method are made for the first system of elements (2.1). For the second system (2.2) only the first approximation is realized. The generating function, the change of variables formulae between averaged and osculating elements, and the right-hand sides of the averaged equations of motion are thus obtained.

\section{Behaviour of the Sun-Jupiter-Saturn system}

The averaged equations are integrated numerically over a time span of 10 Gyr. The equations for slow variables are integrated by 15 order Everhart and 11 order RungeKutta methods. The equations for fast variables are integrated by spline interpolation method.

The accuracy of the integration is controlled by computation of the integrals of energy and area. The absolut value of the relative error for the energy integral calculation is less than $5.2 \cdot 10^{-13}$ for both integrators over a time span of 10 Gyr. The mean value of the relative error is a constant over all integration time.

To calculate the area integrals the properties of the form conservation in Jacobian coordinates (Charlier 1927) and under averaging transform (Kholshevnikov 1991) are used. The area integrals are determined with respect to the Laplace plane. In this case the vector $\sigma=\left(\sigma_{x}, \sigma_{y}, \sigma_{z}\right)$ is directed along the $z$-axis. Hence $\sigma_{x}=\sigma_{y}=0$. The absolute value of the relative error for the $\sigma_{z}$ area integral calculation is $3.5 \cdot 10^{-10}$ for both integrators over a time span of $10 \mathrm{Gyr}$. The amplitude and mean value of the relative error are constant over all integration time. We find out that the area integrals $\sigma_{x}$, $\sigma_{y}$ are preserved with low accuracy: $\left|\sigma_{x} / \sigma_{z 0}\right|<8.2 \cdot 10^{-7},\left|\sigma_{y} / \sigma_{z 0}\right|<5.2 \cdot 10^{-7}$. The non-conservation of the $\sigma_{x}$ and $\sigma_{y}$ integrals has the following reason. As it is proved in Kholshevnikov (1991), they are preserved in the system determined by the averaged Hamiltonian $H$. But they are not preserved in a system determined by a finite sequence of Poisson expansion of the averaged Hamiltonian $H$ taking into account the restrictions (3.3), (3.4) on $k$ and $n$.

Table 1 presents the low and upper limits, mean values and amplitudes of oscillations for the averaged eccentricities and inclinations obtained from the numerical integration of the averaged equations for the first and second approximations over 10 Gyr. The results obtained by the two integrators show a good agreement. The relative differences between the first and second approximations are given in the two last columns of the table 1 . They are more than the small parameter $\mu=1 \cdot 10^{-3}$, but they are generally less than the quotient $\mu / F=4.4 \cdot 10^{-2}$. The only exception is Jupiter's eccentricity amplitude $e_{a}$ for Runge-Kutta method.

The evolution of the ascending node longitudes $\gamma$ of Jupiter and Saturn orbits depends upon the choice of the reference plane. In the first approximation the evolution of the ascending nodes longitudes with respect to the ecliptic plane turns out to be in libration with amplitudes $12.9^{\circ}$ and $32.8^{\circ}$ for Jupiter and Saturn respectively. The libration amplitudes are in agreement with those obtained by Smart (1953). The evolution of the ascending nodes longitudes with respect to the Laplace plane is secular. On the Laplace 
Table 1. Range of the eccentricity and inclination changes for Jupiter and Saturn orbits on time interval 10 Gyr.

\begin{tabular}{|c|c|c|c|c|c|c|}
\hline & \multicolumn{2}{|c|}{ First approximation } & \multicolumn{2}{|c|}{ Second approximation } & \multicolumn{2}{|c|}{ Relative difference } \\
\hline & $\begin{array}{c}15 \text { order } \\
\text { Everhart } \\
\text { method }\end{array}$ & $\begin{array}{c}11 \text { order } \\
\text { Runge-Kutta } \\
\text { method }\end{array}$ & $\begin{array}{c}15 \text { order } \\
\text { Everhart } \\
\text { method }\end{array}$ & $\begin{array}{c}11 \text { order } \\
\text { Runge-Kutta } \\
\text { method }\end{array}$ & $\begin{array}{c}15 \text { order } \\
\text { Everhart } \\
\text { method }\end{array}$ & $\begin{array}{c}11 \text { order } \\
\text { Runge-Kutta } \\
\text { method }\end{array}$ \\
\hline \begin{tabular}{|l|}
$e_{\min }$ \\
$e_{\max }$ \\
$e_{\text {mean }}$ \\
$e_{a}$ \\
\end{tabular} & $\begin{array}{l}0.0184 \\
0.0510 \\
0.0347 \\
0.0163\end{array}$ & $\begin{array}{l}0.0187 \\
0.0508 \\
0.0348 \\
0.0161 \\
\end{array}$ & $\begin{array}{l}\text { J } \\
0.0171 \\
0.0511 \\
0.0341 \\
0.0170\end{array}$ & $\begin{array}{l}\text { Jupiter } \\
0.0171 \\
0.0511 \\
0.0341 \\
0.0170 \\
\end{array}$ & $\begin{array}{l}1.8 \cdot 10^{-2} \\
4.1 \cdot 10^{-2}\end{array}$ & $\begin{array}{l}2.1 \cdot 10^{-2} \\
5.3 \cdot 10^{-2}\end{array}$ \\
\hline \begin{tabular}{|l|}
$i_{\text {min }}$ \\
$i_{\text {max }}$ \\
$i_{\text {mean }}$ \\
$i_{a}$ \\
\end{tabular} & $\begin{array}{l}1.2657 \\
2.0011 \\
1.6334 \\
0.3677 \\
\end{array}$ & $\begin{array}{l}1.2698 \\
2.0002 \\
1.6350 \\
0.3652 \\
\end{array}$ & $\begin{array}{l}1.2705 \\
2.0005 \\
1.6355 \\
0.3650 \\
\end{array}$ & $\begin{array}{l}1.2679 \\
2.0006 \\
1.6342 \\
0.3664 \\
\end{array}$ & $\begin{array}{l}1.3 \cdot 10^{-3} \\
7.4 \cdot 10^{-3}\end{array}$ & $\begin{array}{l}4.9 \cdot 10^{-4} \\
3.3 \cdot 10^{-3}\end{array}$ \\
\hline $\begin{array}{l}e_{\min } \\
e_{\max } \\
e_{\operatorname{mean}} \\
e_{a}\end{array}$ & $\begin{array}{l}0.0212 \\
0.0772 \\
0.0492 \\
0.0280\end{array}$ & $\begin{array}{l}0.0212 \\
0.0771 \\
0.0492 \\
0.0280\end{array}$ & $\begin{array}{l}0.0194 \\
0.0780 \\
0.0487 \\
0.0293\end{array}$ & $\begin{array}{l}\text { Saturn } \\
0.0194 \\
0.0780 \\
0.0487 \\
0.0293\end{array}$ & $\begin{array}{l}1.0 \cdot 10^{-2} \\
4.4 \cdot 10^{-2}\end{array}$ & $\begin{array}{l}1.0 \cdot 10^{-2} \\
4.4 \cdot 10^{-2}\end{array}$ \\
\hline \begin{tabular}{|l|}
$i_{\min }$ \\
$i_{\max }$ \\
$i_{\text {mean }}$ \\
$i_{a}$
\end{tabular} & $\begin{array}{l}0.7240 \\
2.5366 \\
1.6303 \\
0.9063\end{array}$ & $\begin{array}{l}0.7346 \\
2.5319 \\
1.6333 \\
0.8987\end{array}$ & $\begin{array}{l}0.7344 \\
2.5317 \\
1.6331 \\
0.8987\end{array}$ & $\begin{array}{l}0.7340 \\
2.5349 \\
1.6344 \\
0.9005\end{array}$ & $\begin{array}{l}1.7 \cdot 10^{-3} \\
8.5 \cdot 10^{-3}\end{array}$ & $\begin{array}{l}6.7 \cdot 10^{-4} \\
2.0 \cdot 10^{-3}\end{array}$ \\
\hline
\end{tabular}

plane the difference between the ascending nodes longitudes of Jupiter and Saturn orbits is equal to $180^{\circ}$ exactly. This property is used as a test of the integration accuracy.

In the second approximation the evolution of the ascending nodes longitudes with respect to the ecliptic plane turn out to be a large amplitude oscillations with a slow secular motion. The mean values over one period of the ascending node longitudes decrease by $6^{\circ}$ per Gyr.

The evolution of the pericentre longitudes $\beta$ of Jupiter and Saturn orbits turns out to be secular for both approximations.

Table 2 gives estimations of the mean squared norm

$$
\|f\|_{2}=\left(\frac{1}{N} \sum_{j=1}^{N}\left(f_{j}\right)^{2}\right)^{1 / 2}
$$

and the uniform norm

$$
\|f\|_{\infty}=\max _{j=1, \ldots, N}\left|f_{j}\right|
$$

of the variable change functions describing, for each element, the short periodic perturbations, i.e. the differences between mean and osculating elements. Here $N$ is the number of the orbital elements values on the time interval.

The short-period perturbations of Jupiter and Saturn semi-major axes $a$ do not exceed 0.0022 a.u. and 0.0121 a.u. respectively. The tables 1 and 2 comparison shows that the maximum values of the short-period perturbation norms for the eccentricities $e$ and inclinations $i$ are much less than the amplitudes of the corresponding long-period perturbations. The variable change function norms for the longitudes $\alpha, \beta$ and $\gamma$ are much less than the amplitudes of the long-period perturbations also. 
Table 2. Mean squared and uniform norms of the variable change functions.

\begin{tabular}{l|c|c|c|c|c|c|}
\hline \multirow{2}{*}{ Planet } & \multicolumn{7}{c|}{ Orbital elements } \\
\cline { 2 - 7 } & $a$, a.u. & $e$ & $i$, degrees & $\alpha$, degrees & $\beta$, degrees & $\gamma$, degrees \\
\hline \multirow{8}{*}{ Mean squared norm } \\
Jupiter & 0.0006 & 0.0005 & 0.0005 & 0.0113 & 0.9738 & 0.0182 \\
Saturn & 0.0038 & 0.0012 & 0.0013 & 0.0402 & 1.7033 & 0.0522 \\
\hline Jupiter & 0.0022 & 0.0011 & 0.0013 & 0.0304 & 3.1538 & 0.0616 \\
Saturn & 0.0121 & 0.0027 & 0.0032 & 0.1127 & 7.6316 & 0.2603 \\
\hline
\end{tabular}

Estimates of the Liapunov Exponents for the Sun-Jupiter-Saturn system have been obtained. The corresponding Liapunov Time turns out to be 14 Myr (for Jupiter) and 10 Myr (for Saturn).

\section{Conclusion}

A numerical integration of the averaged equations for the Jupiter-Saturn system shows that the motion has a quasiperiodic character over the time-scale of 10 Gyr. The eccentricities and inclinations of Jupiter and Saturn orbits remain small and their values are separated from zero. The lines of nodes and apsides have a secular motion. The shortperiodic perturbations remain small during the considered time interval.

\section{Acknowledgements}

This work was partly supported by the RFBR, Grant 02-02-17516, and the Leading Scientific School, Grant NSh-1078.2003.02.

\section{References}

Brumberg, V. A. 1995, Analytical Techniques of Celestial Mechanics. (Springer, Heidelberg) Charlier, C. L. 1927, Die Mechanik des Himmels. (Walter de Gruyter \& Co., Berlin und Leipzig) Greb, A. V. 2002, PhD thesis, St. Petersburg State University, St. Petersburg

Ivanova, T. V. 1996, in: S.Ferraz-Mello, B.Morando and J.-E.Arlot (eds.), Dynamics, Ephemerides and Astrometry of the Solar System, IAU Symp. 172, (Kluwer Academic Publishers), p. 283

Ivanova, T. 2001, Cel. Mech. Dyn. Astron., 80, 167

Kholshevnikov, K. V. 1991, Astron. Zh., 68, 660

Kholshevnikov, K. V. 1997, Astron. Rep. 41, 135

Kholshevnikov, K. V. 2001 Astron. Rep., 45, 577

Kholshevnikov, K. V., Greb, A. V. and Kuznetsov, E. D. 2001, Solar System Res., 35, 243

Kholshevnikov, K. V. and Greb, A. V. 2001, Solar System Res., 35, 415

Kholshevnikov, K. V., Greb, A. V. and Kuznetsov, E. D. 2002, Solar System Res., 36, 68

Kuznetsov, E. D. and Kholshevnikov, K. V. 2004, Solar System Res., 38, 147

Laskar, J. and Robutel, P. 1995, Cel. Mech. Dyn. Astron., 62, 193

Michtchenko, T. A. and Ferraz-Mello, S. 2001, Icarus, 149, 357

Robutel, P. 1993a, Cel. Mech. Dyn. Astron., 56, 197

Robutel, P. 1993b, Cel. Mech. Dyn. Astron., 57, 97

Robutel, P. 1995, Cel. Mech. Dyn. Astron., 62, 219

Rom A. 1971, Celest. Mech., 3, 331

Smart, W. M. 1953, Celestial mechanics. (Longmans, Green and Co., London, New York, Toronto)

Subbotin, M.F. 1968, Introduction to theoretical astronomy. (Nauka, Moscow) 